\title{
Política de acesso, acessibilidade e inclusão educacional da pessoa com deficiência: revisão integrativa
}

\author{
Policy of access, accessibility and educational inclusion for persons with disabilities: integrative review \\ Política de acceso, accesibilidad e inclusión educativa de personas con discapacidad: revisión integradora
}

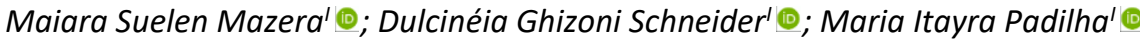

'Universidade Federal de Santa Catarina, Florianópolis, SC, Brasil

\begin{abstract}
RESUMO
Objetivo: conhecer os aspectos abordados pela produção científica em relação à temática da inclusão da pessoa com deficiência nas instituições de ensino. Método: revisão integrativa da literatura nas bases de dados Medical Literature and Retrivial Sistem on Line, Web of science, SCOPUS e Directory of Open Access Journals. Recorte da busca de 2015 a 2019. Resultados: foram analisados vinte e um artigos. As publicações apresentaram temáticas relacionadas à Políticas de acesso, cotas e currículos e Barreiras e facilitadores da inclusão na educação. Considerações finais: os aspectos abordados pela produção científica evidenciaram que entraves são transversais à vivência dos estudantes em suas experiências acadêmicas. Lacunas curriculares, estereotipação do corpo, crenças, falta de capacitação do corpo docente cerca-nos de evidências de que mais do que quebra de paradigmas diante da pessoa com deficiência, é preciso que existam avanços nas práticas políticas para que barreiras como preconceito, discriminação e segregação não definam as relações humanas.

Descritores: Educação; Inclusão Educacional; Política Pública; Política de Educação Superior; Políticas Públicas de não Discriminação.
\end{abstract}

\begin{abstract}
Objective: to learn what concerns are addressed by scientific production on the theme of inclusion for people with disabilities in educational institutions. Method: this integrative literature review searched the Medical Literature Analysis and Retrieval System on Line, Web of Science, SCOPUS and Directory of Open Access Journals database entries from 2015 to 2019. Results: the twenty-one articles examined addressed themes relating to access policies, quotas and curricula, and barriers and facilitators of inclusion in education. Final considerations: the concerns addressed by the scientific production showed that obstacles are transversal to the students' experience in their academic life. Gaps in curricula, body stereotyping, beliefs, lack of capacity-building for teaching staffs surrounded us with evidence that, more than breaking paradigms regarding people with disabilities, there must be advances in political practices, so that barriers such as prejudice, discrimination, and segregation cease to define human relationships.
\end{abstract}

Descriptors: Education; Mainstreaming, Education; Public Policy; Higher Education Policy; Public Nondiscrimination Policies.

\section{RESUMEN}

Objetivo: conocer los aspectos abordados por la producción científica en relación con el tema de la inclusión de personas con discapacidad en las instituciones de enseñanza. Método: revisión integradora de la literatura en las bases de datos Medical Literature and Retrivial Sistem on Line, Web of science, SCOPUS y Directory of Open Access Journals. Recorte de búsqueda de 2015 a 2019. Resultados: se analizaron veintiún artículos. Las publicaciones presentaron temas relacionados con políticas de acceso, cuotas y currículos y Barreras y facilitadores de la inclusión en la educación. Consideraciones finales: los aspectos abordados por la producción científica mostraron que los obstáculos son transversales a la vivencia de los estudiantes en sus experiencias académicas. Lagunas curriculares, estereotipos del cuerpo, creencias, falta de formación del profesorado nos rodean con evidencias de que, más allá de romper paradigmas ante las personas con discapacidad, son necesarios avances en las prácticas políticas para que barreras como el prejuicio, la discriminación y la segregación no definan las relaciones humanas. Descriptores: Educación; Integración Escolar; Política Pública; Política de Educación Superior; Políticas Públicas de no Discriminación.

\section{INTRODUÇÃO}

Acesso e acessibilidade são conceitos que se confundem. $\mathrm{O}$ acesso se refere à ideia de criar condições legais e de direitos igualitários, e a acessibilidade como ações cotidianas e concretas que oferecem oportunidade às pessoas com deficiência de usufruírem os mesmos recursos oferecidos aos demais ${ }^{1}$.

As políticas de inclusão contribuem para o ingresso da educação primária até a universidade. Porém, a matrícula não garante a inclusão educacional nem a acessibilidade, para isso, faz-se necessária uma política institucional de acompanhamento que permita a identificação das necessidades educacionais das pessoas com deficiência².

Agradecimentos ao apoio da Coordenação de Aperfeiçoamento de Pessoal e Nível Superior (CAPES, Brasil) - Código de Financiamento 001, e pela bolsa PROEX/CAPES concedida a Maiara Suelen Mazera - Processo no 88887.481816/2020-00.

Autora correspondente: Maiara Suelen Mazera. E-mail: maiaramazera@gmail.com

Editora Científica: Cristiane Helena Gallasch; Editora Associada: Juliana Amaral Prata 
A ampliação do acesso à Educação Superior na sociedade brasileira, mesmo representando iniciativas importantes no campo da democratização, não é garantia de aprendizagem e não é suficientemente satisfatória para romper com as diferenças excludentes ${ }^{3}$.

O movimento pela inclusão educacional defende o direito de que todos, sem discriminação, devem pertencer, aprender e participar da educação, e as universidades desempenham papel privilegiado de transformação social e aprendizagem ${ }^{2}$.

Com a finalidade de ampliar as condições de permanência dos jovens na educação superior pública federal, o Governo Brasileiro criou em 2005 o Programa Nacional de Assistência Estudantil (PNAES) executado no âmbito do Ministério da Educação. Uma iniciativa complementar foi o Programa de Acessibilidade na Educação Superior, o Programa Incluir, criado em 2005 pela Secretaria de Educação Especial, extinta em 2014, em parceria com a Secretaria de Ensino Superior, ambos vinculados ao Ministério da Educação (MEC). O objetivo principal do Programa Incluir era fomentar a criação e a consolidação de núcleos de acessibilidade nas Instituições Federais de Ensino Superior (IFEs), para efetivar a inclusão de pessoas com deficiência à vida acadêmica, eliminando barreiras comportamentais, pedagógicas, arquitetônicas e de comunicação ${ }^{4}$.

O primeiro desafio que a universidade apresenta aos recém-chegados é o de se tornar um estudante, e para isso depende da continuidade dos estudos e sucesso. Há três tempos sucessivos para a realização desta tarefa: o tempo do estranhamento, o tempo da aprendizagem e o tempo da afiliação ${ }^{5}$. Nos casos de estudantes com algum tipo de deficiência, o percurso até a afiliação apresentará dificuldades suplementares, devendo, portanto, contar com o suporte necessário para responder às novas demandas postas pela universidade.

A inclusão pode ser entendida como um princípio filosófico que preconiza a convivência das diversidades, pressupondo que as diferenças são constituintes do ser humano e se caracterizam como a maior riqueza da vida em sociedade ${ }^{6}$. Uma sociedade inclusiva exige transformações, tanto arquitetônicas e urbanísticas quanto atitudinais diante das pessoas com deficiência, assegurando assim seu acesso, circulação e permanência em espaços comuns ${ }^{7}$. Assim, as instituições de Educação Superior necessitam aperfeiçoar os seus processos de atendimento e acessibilidade, enquanto elementos essenciais para que o estudante permaneça e alcance sucesso em sua aprendizagem ${ }^{8}$.

Essa revisão integrativa da literatura tem como objetivo conhecer os aspectos abordados pela produção científica em relação à temática da inclusão da pessoa com deficiência nas instituições de ensino.

\section{MÉTODO}

Esta revisão integrativa foi realizada no período de 2015 a 2019. O recorte temporal de 2015 referente ao ano da Lei Brasileira de inclusão da pessoa com deficiência, e 2019 que foi o ano de realização desta revisão.

As etapas da revisão integrativa da literatura foram adotadas visando manter o rigor científico: 1) identificação do tema e da questão de pesquisa para a elaboração da revisão integrativa; 2) estabelecimento de critérios para inclusão e exclusão de estudos ou busca na literatura; 3) definição das informações a serem extraídas dos estudos selecionados/categorização dos estudos; 4) avaliação dos estudos incluídos na revisão integrativa; 5) interpretação dos resultados; 6) apresentação da revisão/síntese do conhecimento ${ }^{9}$.

Nesse sentido, para contemplar as etapas da revisão integrativa, inicialmente identificamos a inclusão da pessoa com deficiência nas instituições de ensino como tema de pesquisa, e nos perguntamos "como a produção científica vem abordando a temática da inclusão da pessoa com deficiência nessas instituições?". Posteriormente identificamos publicações indexadas nas bases de dados: Medical Literature and Retrivial Sistem on Line (MEDLINE), Web of science, SCOPUS e Directory of Open Access Journals (DOAJ).

As informações foram coletadas no mês de outubro 2019, obedecendo aos seguintes critérios de inclusão: artigos em português, inglês e espanhol, que estivessem na íntegra; periódicos revisados por pares, incluídos no período de 2015 a 2019 que continham em seus títulos e/ou resumos os seguintes descritores baseados nos Descritores em Ciências da Saúde (DeCS): "Mainstreaming Education OR Inclusão educacional AND Disabled Persons OR Pessoas com deficiência AND Universities OR Universidades". Foram consideradas as seguintes categorias de artigos: pesquisa, reflexão e revisões de literatura. Como critério de exclusão, adotamos artigos que não contemplassem a temática proposta.

A partir disto, para contemplar a terceira etapa, organizamos e sumarizamos as informações de maneira concisa, formando um banco de dados de fácil acesso e manejo com uso de um software de gerenciamento bibliográfico EndNote. Foi realizada a leitura dos estudos conforme representada no fluxograma PRISMA na Figura 1. 

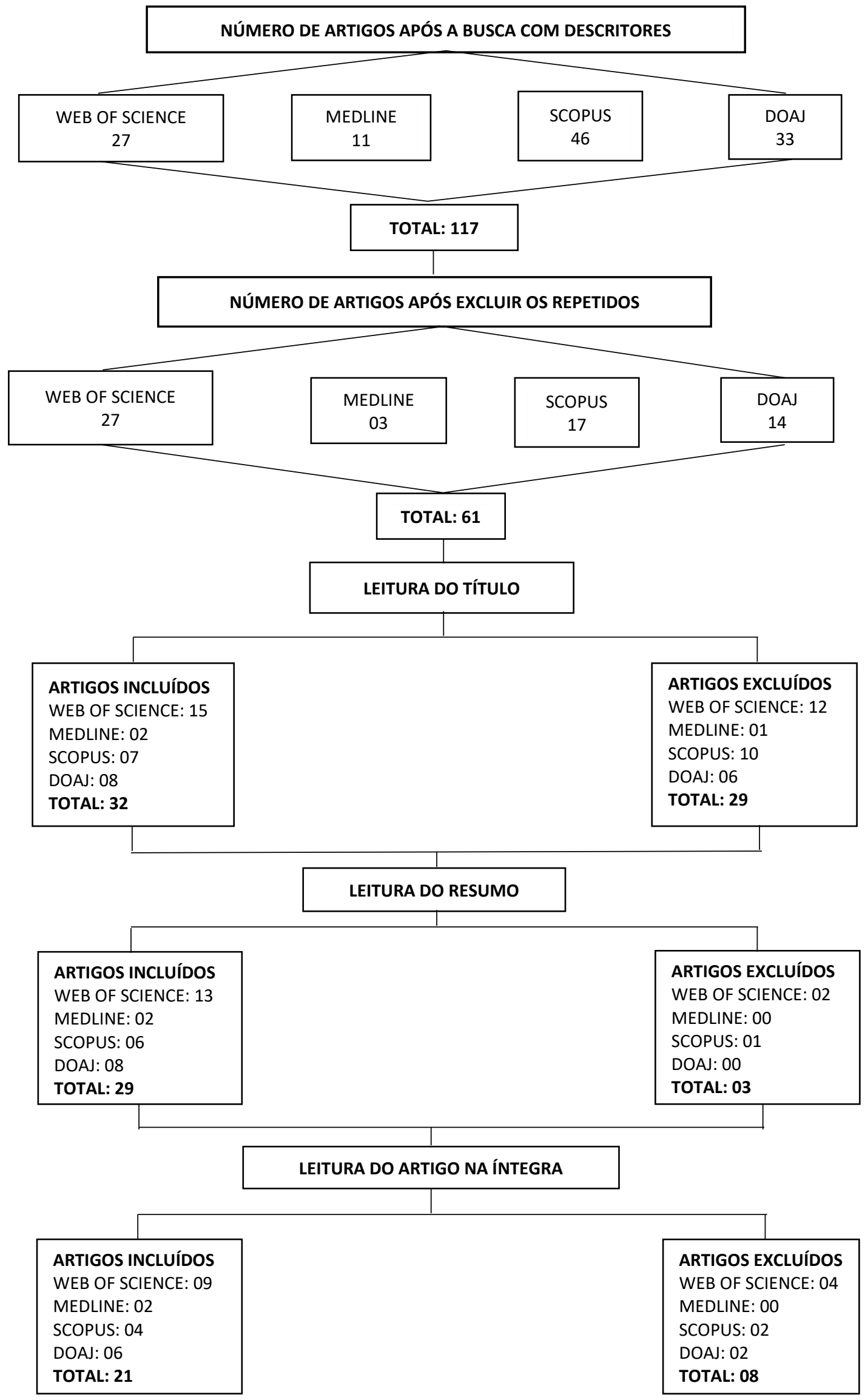

FIGURA 1: Fluxograma PRISMA que orientou a busca pelos artigos, Florianópolis, SC, Brasil, 2019. 
Para contemplar a etapa quatro da revisão integrativa realizamos uma análise detalhada dos artigos selecionados, procurando os resultados diferentes ou conflitantes nos estudos selecionados. A quinta etapa envolveu a fase da discussão dos resultados, fundamentada na avaliação crítica dos estudos incluídos e em comparação com o conhecimento teórico de estudos nacionais e internacionais. Nessa etapa, a construção das categorias de análise emergiram das leituras flutuantes dos textos. Na última etapa da revisão, apresentamos uma síntese do conhecimento materializada por meio deste artigo científico.

\section{RESULTADOS}

Foram encontrados nove artigos na base indexadora Web Of Science, dois artigos na MEDLINE, quatro artigos na SCOPUS e seisa artigos na base DOAJ (Figura 2).

\begin{tabular}{|c|c|c|c|}
\hline Título & Ano & Periódico & País \\
\hline $\begin{array}{l}\text { Inclusive education a "rhetoric" or "reality"? Teachers' perspectives } \\
\text { and beliefs" }{ }^{10} \text {. }\end{array}$ & 2015 & Teaching and Teacher Education & EUA \\
\hline $\begin{array}{l}\text { Social participation of children and youth with disabilities in Canada, } \\
\text { France and Germany }{ }^{11} \text {. }\end{array}$ & 2015 & $\begin{array}{l}\text { International Journal of Inclusive } \\
\text { Education }\end{array}$ & Canadá \\
\hline $\begin{array}{l}\text { Bullying and Students with Disabilities: Examination of Disability Status } \\
\text { and Educational Placement }{ }^{12} \text {. }\end{array}$ & 2015 & School Psychology Review & EUA \\
\hline $\begin{array}{l}\text { A deficiência em foco nos currículos de graduação da UFRN: uma } \\
\text { abordagem histórica (1960-2015) }\end{array}$ & 2016 & Holos & Brasil (RN) \\
\hline $\begin{array}{l}\text { Legislação para estudantes com deficiência no ensino superior no } \\
\text { Brasil e em Portugal: algumas reflexões }{ }^{14} \text {. }\end{array}$ & 2016 & Acta Scientiarum. Education & Brasil (PR) \\
\hline $\begin{array}{l}\text { Análise de Acessibilidade: Aplicação Parcial do Modelo Sicogea em um } \\
\text { Centro de Ensino de uma Instituição Federal de Ensino }{ }^{15} \text {. }\end{array}$ & 2016 & $\begin{array}{l}\text { Revista de Gestão Ambiental e } \\
\text { Sustentabilidade }\end{array}$ & Brasil (SC) \\
\hline $\begin{array}{l}\text { Disability inclusion in higher education in Uganda: Status and } \\
\text { strategies }^{16} \text {. }\end{array}$ & 2016 & Revista Africana de Deficiência & Africa (Uganda) \\
\hline Inclusive education in New Zealand: rhetoric and reality ${ }^{17}$. & 2016 & $\begin{array}{l}\text { History of } \\
\text { Education Review }\end{array}$ & Nova Zelândia \\
\hline A inclusão na prática: alunos com deficiência no Ensino Superior ${ }^{18}$. & 2017 & $\begin{array}{l}\text { Revista Eletrônica Política e Gestão } \\
\text { Educacional }\end{array}$ & Brasil (SP) \\
\hline $\begin{array}{l}\text { Endrew F. v. Douglas County School District (2017) and special } \\
\text { education law: What teachers and administrators need to know }{ }^{19} \text {. }\end{array}$ & 2017 & $\begin{array}{l}\text { Preventing School Failure: } \\
\text { Alternative Education for } \\
\text { Children and Youth }\end{array}$ & EUA \\
\hline $\begin{array}{l}\text { Students with Disabilities: Practical Information for School Counselors } \\
\text { in Turkey }{ }^{20} \text {. }\end{array}$ & 2017 & Int J Adv Counselling & Turquia e USA \\
\hline $\begin{array}{l}\text { The role of entrepreneurship education in shaping entrepreneurial } \\
\text { action of disabled students in Nigeria } 21 .\end{array}$ & 2017 & $\begin{array}{l}\text { Journal of Small Business \& } \\
\text { Entrepreneurship }\end{array}$ & Nigéria e África \\
\hline $\begin{array}{l}\text { The Impact of a Faculty Training Program on Inclusive Education and } \\
\text { Disability } 22 \text {. }\end{array}$ & 2017 & $\begin{array}{l}\text { Evaluation and Program } \\
\text { Planning }\end{array}$ & Espanha \\
\hline $\begin{array}{l}\text { Attitudes towards inclusion in higher education in a Portuguese } \\
\text { university²3. }\end{array}$ & 2017 & $\begin{array}{l}\text { International Journal of Inclusive } \\
\text { Education }\end{array}$ & Portugal \\
\hline $\begin{array}{l}\text { Including students with disabilities in Education for All: lessons from } \\
\text { Ethiopia }{ }^{24} \text {. }\end{array}$ & 2017 & $\begin{array}{l}\text { International Journal of Inclusive } \\
\text { Education }\end{array}$ & EUA \\
\hline $\begin{array}{l}\text { Oferta, Demanda e Nota de Corte: Experimento Natural sobre Efeitos } \\
\text { da Lei das Cotas no Acesso à UFMG }{ }^{25} \text {. }\end{array}$ & 2018 & $\begin{array}{l}\text { DADOS-Revista de Ciências } \\
\text { Sociais }\end{array}$ & Brasil (MG) \\
\hline $\begin{array}{l}\text { Facilitators and Barriers of Assistive Technology and Learning } \\
\text { Environment for Children with Special Needs }{ }^{26} \text {. }\end{array}$ & 2018 & $\begin{array}{l}\text { Occupational Therapy } \\
\text { International }\end{array}$ & Tailândia \\
\hline La inclusión en la formación inicial de profesores de matemáticas ${ }^{27}$. & 2018 & $\begin{array}{l}\text { Acta Scientiarum. Education, [s.l.], } \\
\text { v. } 40, \text { n. } 3, \text { p.1-12 }\end{array}$ & Brasil (PR) \\
\hline $\begin{array}{l}\text { Implementation of disability policy framework in Namibia: A } \\
\text { qualitative study }{ }^{28} \text {. }\end{array}$ & 2018 & Revista Sul-Africana de Fisioterapia & África do Sul \\
\hline $\begin{array}{l}\text { Funding and inclusion in higher education institutions for students } \\
\text { with disabilities }{ }^{29} \text {. }\end{array}$ & 2019 & Revista Africana de Deficiência & África do Sul \\
\hline $\begin{array}{l}\text { Image is everything: educator awareness of perceived barriers for } \\
\text { students with physical disabilities in geoscience degree programs }{ }^{30} \text {. }\end{array}$ & 2019 & $\begin{array}{l}\text { Journal Of Geography In Higher } \\
\text { Education }\end{array}$ & EUA \\
\hline
\end{tabular}

FIGURA 2: Artigos selecionados para a revisão. Florianópolis, SC, Brasil, 2019. Fonte: Autoras, 2019. 
Referente à quantidade de artigos e seus locais de publicação, no Brasil tivemos o quantitativo de seis artigos publicados nos Estados Unidos da América, quatro no Continente Africano, um no Canadá, uma na Tailândia, um na Nova Zelândia, um em Portugal e um na Espanha.

Com base no exposto, discutiremos os achados referentes aos aspectos abordados em relação à temática da inclusão educacional da pessoa com deficiência por meio de duas categorias: Políticas de acesso, cotas e currículos; e Barreiras e facilitadores da inclusão educacional.

\section{DISCUSSÃO}

\section{Políticas de acesso, cotas e currículos}

A inclusão social e educacional da pessoa com deficiência assume um lema ético e moral, e um dever social, no sentido de sanar as divergências de assistência às suas demandas cotidianas.

A inclusão social e educacional é um paradigma que garante ao aluno com deficiência o acesso e permanência ao sistema educacional qualificado. As pessoas com alguma deficiência estão menos presentes na educação regular quando comparadas àquelas sem deficiência, percentualmente ${ }^{31}$. Esse percentual é importante, pois gera no futuro uma grande diferença no quantitativo de pessoas com deficiência inseridas no ensino superior.

A publicação de normas jurídicas tem sido uma medida importante para garantir os direitos dessa população e combater, de forma mais incisiva, a discriminação e o preconceito em face à condição da deficiência ${ }^{14}$.

Mundialmente, a El ganhou notoriedade a partir de 1994, por meio da declaração elaborada na Conferência Mundial sobre Educação Especial, realizada em Salamanca. Essa Declaração relatou a importância da inclusão educacional das pessoas com deficiência, defendendo que as instituições de ensino se adequassem às necessidades dos indivíduos nelas matriculados e estabeleceu um acordo entre países e organizações internacionais que se comprometeram a promover mudanças na educação para atender todos os estudantes independentemente de suas diferenças ou dificuldades ${ }^{14,18,32}$.

O Brasil se comprometeu a efetuar políticas públicas para inserir as pessoas com deficiência na sociedade, passando a editar leis, como a Lei de Diretrizes e Bases da Educação Nacional a LDB (Lei no 9.394/1996) aprovada em dezembro de $1996^{14}$.

Nos anos 1970 e 1980, Portugal iniciou uma progressiva consagração dos direitos fundamentais dos cidadãos com deficiência, expressa em dispositivos legais como: a Constituição da República Portuguesa em 1976, a Lei de Bases do Sistema Educativo (Lei n.o 46 de 1986) e a Lei de Bases da Prevenção e da Reabilitação e Integração das Pessoas com Deficiência (Lei n.o 9/1989) ${ }^{14}$. Percebe-se que Portugal esteve mais avançado que o Brasil quando se trata de assegurar direitos às pessoas com deficiência, o que pode justificar o atuante trabalho do país na área da reabilitação nos dias atuais. A legislação sobre inclusão para pessoas com deficiência no Ensino Superior no Brasil e em Portugal se encontra em estágios diferenciados, o que parece ser perfeitamente compreensível, partindo do entendimento da El enquanto processo ${ }^{14}$.

Em um estudo ${ }^{2}$ realizado na Namíbia evidenciou que, apesar da existência de um programa de El organizado pelo Ministério da Educação em nível nacional com foco em estudantes com deficiência auditiva e visual, persistem lacunas nos direitos humanos de pessoas com deficiência por se tratar de um país com muitas fragilidades sociais, econômicas e políticas. Ainda, no tocante à educação primária e secundária, foi abordada a falta de equidade na El e de preparação adequada prévia ao lançamento da Política Setorial sobre a educação inclusiva ${ }^{28}$

No Reino Unido, a inclusão de pessoas com deficiência nas IES é atualmente facilitada pela Lei da Igualdade de 2010, a qual proíbe a discriminação contra pessoas com deficiência em todas as esferas de suas vidas. No âmbito do ensino superior, o modelo de financiamento para pessoas com deficiência baseia-se, principalmente, no princípio da igualdade no acesso à educação ${ }^{29}$. Esses aspectos de inclusão realizados pelo país corroboram o seu desenvolvimento, tendo em vista que é um Estado soberano e que reconhece a pessoa com deficiência como capaz de exercer funções na sociedade.

Nos Estados Unidos da América, o Congresso promulgou a Lei de Reabilitação, em 1973, como uma medida para proibir a discriminação contra pessoas com deficiência, criando incentivos na forma de financiamento federal às IES que tivessem suas instalações mais acessíveis, como salas de aula adaptadas. Essa lei desempenhou um papel importante no aprimoramento da inclusão desses estudantes, proibindo a sua discriminação ${ }^{29}$. Similarmente, o Canadá adotou uma abordagem de direitos humanos para promover a inclusão de estudantes com deficiência, com o objetivo de dar oportunidades iguais e proibir a discriminação ${ }^{29}$.

$\mathrm{Na}$ Índia, os avanços no acesso e na equidade estão ameaçados pelo constante declínio do financiamento das IES pelo governo indiano desde meados da década de 1990, o que resultou em um ensino superior cada vez mais financiado por fundos não governamentais, perpetuando a exclusão e a marginalização desses estudantes ${ }^{29}$. 
Os alunos com deficiência em Portugal têm garantia à igualdade de acesso ao ensino superior, porém a fragilidade se encontra em não ter acompanhamento desses alunos para garantir a equidade durante o seu desenvolvimento acadêmico ${ }^{23}$.

No Brasil, foi promulgada em 2012 e, gradualmente, implementada a partir de 2013 a Lei das Cotas ${ }^{33}$ (Lei no 12.711, de 29 de agosto de 2012), que estabeleceu um padrão mínimo para as ações afirmativas no acesso ao ensino superior federal. Em 2016, a Lei n. 13.409, de 28 de dezembro, alterou a Lei de Cotas incluindo a reserva de vagas para pessoas com deficiência ${ }^{34}$. Neste contexto, verifica-se o incremento da demanda brasileira pelo ensino superior, culminando na mudança do perfil discente, onde a permanência tende a ser maior entre cotistas, com desempenho acadêmico similar ao de outros estudantes ${ }^{25}$.

Referente ao currículo dos cursos de graduação, a educação é a área de conhecimento que abrange o maior número de componentes curriculares que versam sobre a temática da deficiência, correspondendo a $44 \%{ }^{13}$. Em seguida, seguem os componentes curriculares referentes ao ensino de libras, totalizando $38 \%$. A saúde, as tecnologias e a música albergam 9\%, 5,5\% e 3\%, respectivamente. Esse estudo apontou que há um crescimento na quantidade de componentes curriculares que versam sobre a deficiência nos currículos dos cursos de graduação. Entretanto, este número restringese às licenciaturas, não oportunizando aos alunos dos demais cursos a discussão e aprofundamento da deficiência inserida nos diversos modos de viver e $\operatorname{agir}^{13}$. Na área da saúde a lacuna permanece, pois os currículos ainda são apoiados no modelo biomédico e curativista.

Neste sentido, mudanças curriculares abrangentes para uma educação inclusiva, mostram-se essenciais e requerem a implementação de ações formativas para professores ${ }^{30}$, bem como a identificação de barreiras à participação dos alunos com deficiências para melhorar a acessibilidade. Em outra perspectiva, também se mostra necessário proporcionar aos futuros profissionais conhecimentos sobre a inclusão de pessoas com deficiência, a fim de contribuir com reflexões sobre a prática pedagógica frente às diferenças e promover uma educação e atendimento verdadeiramente inclusivos ${ }^{27}$.

$\mathrm{O}$ acesso ao ensino fundamental, médio e superior possibilitam o desenvolvimento da pessoa com deficiência e contribuem com reflexões para a concretização da democracia e justiça, desde que oferecidos com adaptações e em atendimento às distintas necessidades. No entanto, o alcance destas questões se depara com o distanciamento pelo assunto que, motivado pelo preconceito, resulta na reprodução de conceitos e paradigmas ultrapassados, centrados nos modelos de deficiência caritativo e médico

\section{Barreiras e facilitadores da inclusão educacional}

O maior problema enfrentado pelas pessoas com deficiência não é a ausência de leis, tampouco legislações aplicáveis aos casos concretos como observamos na categoria anterior, pois o grande impasse reside na sua aplicabilidade.

Entendemos que igualmente importante ao estabelecimento de leis que possibilitam o ingresso e a permanência dos estudantes, também é indispensável a implementação da formação continuada de docentes nas diretrizes do ensino fundamental, médio e superior. A preparação do professor para atender aos alunos com deficiência é primordial para que o processo ensino-aprendizagem seja concretizado e que potencialize uma educação inclusiva ${ }^{14}$. Diante disto, é necessário ir além da luta pelos direitos e buscar, de fato, instrumentos e recursos para uma educação que promova o desenvolvimento ao máximo.

Nas últimas décadas muitos países conseguiram implementar políticas favoráveis à inclusão de estudantes com deficiência na educação geral. Contudo, em outros países, o quadro político não é bem-sucedido. Em um estudo realizado em Nova Delhi ${ }^{10}$, capital da Índia, os professores definiram que estudantes com deficiência são aqueles que têm deficiências físicas ou intelectuais. Embora essa atitude demonstre o apoio dos docentes aos estudantes com deficiência, ela revela uma visão deficitária de como incluí-los, haja vista a ausência de normativas institucionais.

Nesta mesma investigação, constatou-se que muitos professores vislumbram a inclusão como um conceito baseado nos princípios de moralidade e ética, pois as práticas são influenciadas por seus sistemas de crenças e normas. Tais percepções se mostraram por meio do uso de exemplos religiosos para justificar a sua prática da inclusão e da posição contrária à inclusão de estudantes com deficiência em salas de aula comuns, por considerá-los especiais, vulneráveis, fracos e, por isso, mais suscetíveis ao bullying ${ }^{10}$.

Estudo realizado nos Estados Unidos da América afirma que os alunos com deficiência se envolvem de modo desproporcional em bullying e em lutas com seus pares sem deficiência, sugerindo que estes estudantes sofrem mais vitimização ${ }^{12}$. Como barreiras para uma prática educativa inclusiva bem-sucedida, os professores de Nova Delhi citaram a humilhação de estudantes com deficiência por seus pares ou professores, pois os alunos com deficiência não são capazes de se defender das humilhações. 
$\mathrm{Na}$ Índia, apesar do castigo corporal ser banido pelos sistemas legislativo e judicial, continua a ser um método disciplinar popular nas escolas. Outras barreiras citadas foram fatores sistemáticos, como as turmas grandes e a falta de preparo para atender alunos com deficiência ${ }^{10}$. Metade dos professores de Nova Delhi considerou a deficiência uma condição que requer uma abordagem de tratamento médico e não um modelo educacional ${ }^{10}$

Além disso, considerando que os estudantes são estimulados a seguir vocações, frequentemente, alunos com deficiência visual são vistos como cantores ${ }^{10}$. A estereotipação da pessoa com deficiência também apareceu em uma pesquisa $^{30}$ realizada no Reino Unido, quando perguntado sobre a percepção de acesso e inclusão de estudantes com deficiência física nos cursos de geociências, a resposta dos professores foi que o curso é visto como uma ciência para pessoas capazes, que escalam montanhas e vão para locais remotos.

A definição da deficiência muda com base no modelo adotado, a Organização Mundial de Saúde (OMS) aponta a deficiência como uma interação entre condições de saúde, fatores ambientais e fatores pessoais ${ }^{35}$. Da mesma forma, a Classificação Internacional de Saúde e Funcionalidade (CIF) fornece uma classificação universal de deficiência e saúde, conceitua deficiência como termo genérico para deficiências, restrições de atividades e limitações de participação ${ }^{36}$.

Essas conceituações de deficiência destacam a necessidade de conhecer o indivíduo como um todo, bem como as suas limitações, restrições e experiências como resultado de fatores biológicos, psicológicos e sociais. Por isso, professores e coordenadores de escolas devem estar cientes das diferenças individuais ao trabalhar com alunos com deficiência, porque esses alunos não têm as mesmas experiências, limitações ou respostas à sua diferença, mesmo quando eles têm o mesmo tipo de deficiência ${ }^{20}$.

O impacto positivo da capacitação em deficiência e El na prática do ensino foi demonstrado em um estudo sobre o conhecimento adquirido por 20 professores após sua participação em um programa de treinamento sobre El e deficiência em uma Universidade da Espanha, destacando que os mesmos se perceberam mais informados, treinados e conscientes ${ }^{22}$.

Para a concretização da El, é necessário que professores, pais e comunidade acreditem que todos os estudantes com deficiência podem aprender ${ }^{24}$. Nesse sentido, os educadores devem estar cientes das leis e atualizações relacionadas à El, conhecer as melhores práticas a serem implementadas ${ }^{19}$, pois investir em educação também consiste em capacitar docentes para realizar uma El, sendo fundamental desmistificar crenças e modelos de deficiência ultrapassados.

\section{Limitações do estudo}

Destaca-se, como limitação deste estudo, a ausência de obras da literatura cinzenta que poderiam trazer mais elementos discutíveis para a avaliação dessa revisão integrativa da literatura.

\section{CONSIDERAÇÕES FINAIS}

É possível presenciar o quanto estamos longe de uma igualdade de direitos em diversos segmentos da sociedade. Embora muito tenha sido alcançado sobre a El no ensino básico, médio e superior, em termos práticos, mantêm-se longe dos pressupostos teóricos e normativos.

Os aspectos abordados pela produção científica em relação à temática da inclusão da pessoa com deficiência nas instituições de ensino revelam que muitos países em desenvolvimento têm leis e normativas para implementar a El nas escolas e universidades, as quais foram criadas a partir de um grande acordo mundial.

Quando se fala em inclusão, acesso e acessibilidade na educação não se pode tratar apenas das questões de estrutura e acesso físico, mas sim, de como acompanhar a pessoa com deficiência, promovendo condições para o seu desenvolvimento e para a construção de conhecimentos. Como vimos nos achados dessa pesquisa, as leis existem, mas não proporcionam a permanência dos estudantes com deficiência nas instituições de ensino, muitos entraves são transversais à vivência desses estudantes em suas experiências acadêmicas.

Referente aos obstáculos presentes na educação dos estudantes com deficiência, os estudos apresentaram a escassez de financiamento, as fragilidades referentes à temática da deficiência e inclusão nos currículos de graduação, a estereotipação do corpo com limitação, as crenças limitantes e falta de capacitação dos professores para realizar a inclusão do aluno com deficiência desde o ensino básico ao ensino superior.

Os estudos dessa revisão nos cercaram de evidências de que mais do que quebrar paradigmas diante da pessoa com deficiência, é preciso que existam avanços nas práticas políticas para que barreiras como preconceito, discriminação e segregação não definam as relações humanas.

Referente às implicações das análises no âmbito da Enfermagem, esta revisão da literatura expõe a importância de estudos como este na formação dos enfermeiros considerando que no currículo acadêmico desses profissionais há uma lacuna referente às questões de deficiência e inclusão. Tendo em vista que a temática é transversal aos conteúdos, sua abordagem se faz necessária, pois o enfermeiro compreende a influência dos 
determinantes sociais na saúde dos indivíduos e participa ativamente do processo de reabilitação e ressocialização das pessoas com deficiência.

\section{REFERÊNCIAS}

1. Manzini EJ. Acessibilidade: um aporte na legislação para o aprofundamento do tema na área da educação. In: Baptista CR. et al. (Orgs.). Educação Especial: diálogo e pluralidade. Porto Alegre: Mediação, 2008.

2. Vieira NJW, Severo A, Albertani J. Inclusão no Ensino Superior: acesso e permanência dos alunos com deficiência na Universidade Federal de Santa Maria no período de 2010 a 2012. In: Pieczkowski TMZ, Naujorks MI (Org.). Educação, inclusão e acessibilidade: diferentes contextos. Chapecó: Argos, 2014. p. 21-44.

3. Pavão SMO, Fiorin RPA. O uso da agenda como estratégia psicopedagógica na educação superior. In: Pavão, SMO, et al (Org.). Aprendizagem e acessibilidade: Travessias do aprender na Universidade. Santa Maria: Pró-reitoria de Extensão (pre), 2015. p. 116-128. [cited 2019 Jun 14]; Available from: http://coral.ufsm.br/anima/images/Livro\%201.pdf.

4. Brasil. Ministério da Educação. Documento orientador Programa Incluir - Acessibilidade na educação superior SECADI/SESU. 2013. [cited 2019 Jun 14]; Available from:

http://portal.mec.gov.br/index.php?option=com_docman\&view=download\&alias=13292-doc-oriprogincl\&category_slug=junho-2013-pdf\&Itemid=30192.

5. Coulon A. A condição de estudante: a entrada na vida universitária. Salvador: Edufba, 2008.

6. Oliveira AAS, Leite LP. Construção de um sistema educacional inclusivo: um desafio político-pedagógico. Ensaio: Avaliação e Políticas Públicas em Educação. [Internet]. 2007 [cited 2019 Jun 14]; 15(57):511-24. DOI: http://dx.doi.org/10.1590/s010440362007000400004

7. Martins SEO, Gomes AJV, Fernandes YZ, Benetti CS. Inclusão de universitários com deficiência na educação superior: o que dizem as pesquisas no Brasil e Uruguai. Jornal de Políticas Educacionais. [Internet]. 2017 [cited 2019 Jun 15]; 11(17). DOI: http://dx.doi.org/10.5380/jpe.v11i0.53635.

8. Fantacini RAF, Almeida MA. Systematic review on the presence of Accessibility Nucleus in Higher Education EaD - 2005 to 2018. Revista Educação Especial. [Internet] 2019 [cited 2019 Jun 15]; 32. DOI: http://dx.doi.org/10.5902/1984686X36305.

9. Mendes KD, Silveira RCCP, Galvão CM. Integrative literature review: a research method to incorporate evidence in health care and nursing. Texto Contexto Enferm. [Internet] 2008 [cited 2019 Ago 02]; 17(4);758-64. DOI: https://doi.org/10.1590/S010407072008000400018.

10. Tiwari A, Das A, Sharma M. Inclusive education a "rhetoric" or "reality"? Teachers' perspectives and beliefs. Teaching and Teacher Education. [Internet] 2015 [cited 2019 Ago 04]; 52;128-136. DOI: http://dx.doi.org/10.1016/j.tate.2015.09.002.

11. Schneider C. Social participation of children and youth with disabilities in Canada, France and Germany. International Journal of Inclusive Education. [Internet] 2015 [cited 2019 Ago 02]; 19(10):1068-79. DOI: https://doi.org/10.1080/13603116.2015.1037867.

12. Rose CA. et al. Bullying and Students with Disabilities: Examination of Disability Status and Educational Placement. School Psychology Review. National Association of School Psychologists (NASP). [Internet] 2015 [cited 2019 Ago 03]; 44(4):425-44. DOI: https://doi.org/10.17105/spr-15-0080.1.

13. Amorim EG, Medeiros Neta OM, Guimarães JÁ. Legislation for higher education disabled students in Brazil and Portugal: some reflections. HOLOS. [Internet] 2016 [cited 2019 Ago 04] 32[2]. DOI: https://doi.org/10.15628/holos.2016.4000.

14. Melo FRLV, Martins MH. Legislation for higher education disabled students in Brazil and Portugal: some reflections. Acta Scientiarum. Education, [Internet] 2016 [cited 2019 Feb 02]; 38(3):259-69. DOI: https://doi.org/10.4025/actascieduc.v38i3.30491.

15. Freitas KE, Pfitscher ED, Belan AB. Accessibility analysis: application of partial sicogea model in a training center of a federal institution of education. Rev. Gest. Ambient. Sustentabilidade [Internet] 2016 [cited 2019 Feb 03]; 5(3). Available from: http://www.revistageas.org.br/ojs/index.php/geas/article/view/394.

16. Emong P, Eron L. Disability inclusion in higher education in Uganda: Status and strategies. Afr J Disabil. [Internet] 2016 [cited 2019 Jun 20]; 5(1):193. DOI: http://dx.doi.org/10.4102/ajod.v5i1.193.

17. Selvaraj JA. Inclusive education in New Zealand: rhetoric and reality. History Of Education Review. [Internet] 2016 [cited 2019 Jun 21]; 45(1):54-68. DOI: http://dx.doi.org/10.1108/her-04-2014-0029.

18. Wellichan DSP, Souza CS. Inclusion in practice: students with disabilities in higher education. Revista Eletrônica Política e Gestão Educacional. [Internet] 2017 [cited 2019 Jun 21]; 21(1):146-66. DOI: http://dx.doi.org/10.22633/rpge.v21.n1.2017.9786.

19. Couvillon MA, Yell ML, Katsiyannis A. Endrew F. v. Douglas County School District (2017) and special education law: What teachers and administrators need to know. Preventing School Failure: Alternative Education for Children and Youth, [Internet] 2018 [cited 2019 Ago 08]; 62(4):289-99. DOI: http://dx.doi.org/10.1080/1045988x.2018.1456400.

20. Cimsir E, Hunt B. Students with Disabilities: Practical Information for School Counselors in Turkey. International Journal for the Advancement of Counselling. [Internet] 2017 [cited 2019 Ago 09]. DOI: https://doi.org/10.1007/s10447-017-9315-x.

21. Dakung RJ, et al. The role of entrepreneurship education in shaping entrepreneurial action of disabled students in Nigeria. Journal Of Small Business \& Entrepreneurship. [Internet] 2017 [cited 2019 Ago 13]; 29(4):293-311. DOI: http://dx.doi.org/10.1080/08276331.2017.1312217.

22. Moriña A, Carballo R. The impact of a faculty training program on inclusive education and disability. Evaluation and Program Planning. [Internet] 2017 [ cited 2019 Ago 13]; 65:77-83. DOI: http://dx.doi.org/10.1016/j.evalprogplan.2017.06.004. 
23. Martins MH, Borges, ML, Gonçalves T. Attitudes towards inclusion in higher education in a Portuguese university. International Journal Of Inclusive Education. [Internet] 2017 [cited 2019 Jun 13]; 22(5):527-42. DOI: http://dx.doi.org/10.1080/13603116.2017.1377299.

24. Franck B, Joshi DK. Including students with disabilities in Education for All: lessons from Ethiopia. International Journal Of Inclusive Education. [Internet] 2016 [cited 2019 Jun 04]; 21(4):347-60. DOI: http://dx.doi.org/10.1080/13603116.2016.1197320.

25. Karruz A. Supply, demand and cut-off note: natural experiment on effects of the Quotas Law on access to the Federal University of Minas Gerais. Dados. [Internet] 2018 [cited 2019 Jun 13]; 61(2):405-62. DOI: http://dx.doi.org/10.1590/001152582018157.

26. Lersilp S, Putthinoi S, Lersilp T. Facilitators and Barriers of Assistive Technology and Learning Environment for Children with Special Needs. Occupational Therapy International. [Internet] 2018 [cited 2019 Jun 20]; 1(9). DOI: https://doi.org/10.1155/2018/3705946.

27. Silva, SCR, Viginheski LVM, Shimazaki EM. Inclusion in the initial training of teachers of mathemati. Acta Scientiarum. Education. [Internet] 2018 [cited2019 Jun 13]; 40(3):1-12. DOI: https://doi.org/10.4025/actascieduc.v40i3.32210.

28. Shumba TW, Moodley I. Implementation of disability policy framework in Namibia: A qualitative study. S Afr J Physiother. [Internet] 2018 [cited 2019 Jun 13]; 74(1):400. DOI: https://doi.org/10.4102/sajp.v74i1.400.

29. Chiwandire $D$, Vincent $L$. Funding and inclusion in higher education institutions for students with disabilities. African Journal of Disability. [Internet] 2019 [cited 2019 Jun 15]; 8. DOI: http://dx.doi.org/10.4102/ajod.v8i0.336.

30. Mol L, Atchison C. Image is everything: educator awareness of perceived barriers for students with physical disabilities in geoscience degree programs. Journal Of Geography In Higher Education. [Internet] 2019 [cited 2019 Jun 13]; 43(4):544-67. DOI: https://doi.org/10.1080/03098265.2019.1660862.

31. Instituto Brasileiro De Geografia e Estatística. Pesquisa Nacional de Saúde: 2013: Ciclos de Vida: Brasil e Grandes Regiões/ IBGE, Coordenação de Trabalho e Rendimento. Rio de Janeiro: IBGE, 2015. 92 p.

32. Burci TVL, Costa MLF. Inclusion of people with visual impairment in distance education. Acta Scientiarum. Education. [Internet] 2018 [cited 2019 Jun 16]; 40(2):e32212. DOI: https://doi.org/10.4025/actascieduc.v40i2.32212.

33. Brasil. Lei no 12.711, de 29 de agosto de 2012. Dispõe sobre o ingresso nas universidades federais e nas instituições federais de ensino técnico de nível médio e dá outras providências. 2012. [cited 2019 Jun 16]. Available from: http://www.planalto.gov.br/ccivil_03/_ato2011-2014/2012/lei/l12711.htm.

34. Brasil. Lei no 13.409, de 28 de dezembro de 2016. Altera a Lei no 12.711, de 29 de agosto de 2012, para dispor sobre a reserva de vagas para pessoas com deficiência nos cursos técnico de nível médio e superior das instituições federais de ensino. 2016. [cited 2019 Jun 17]. Available from: http://www.planalto.gov.br/ccivil_03/_ato2015-2018/2016/lei//13409.htm.

35. Organização Mundial da Saúde. Relatório Mundial sobre a Deficiência / World Health Organization, The World Bank; Tradução Lexicus Serviços Linguísticos. São Paulo: SEDPcD. [Internet] 2012. [cited 2019 Jun 17]. 334 p. Available from: https://apps.who.int/iris/bitstream/handle/10665/44575/9788564047020_por.pdf?sequence=4.

36. Organização Mundial da Saúde. Classificação Internacional da Funcionalidade, Incapacidade e Saúde. [Internet] 2001 [cited 2019 Jun 17]. Available from: http://www.periciamedicadf.com.br/cif2/cif_portugues.pdf. 